\title{
Fetal growth in early pregnancy and risk of delivering low birth weight infant: prospective cohort study
}

\author{
Radek Bukowski, associate professor, ${ }^{1}$ Gordon C S Smith, professor, ${ }^{2}$ Fergal D Malone, professor, ${ }^{3}$ Robert H \\ Ball, associate professor, ${ }^{4}$ David A Nyberg, director, ${ }^{5}$ Christine H Comstock, director of fetal imaging, ${ }^{6}$ Gary D \\ V Hankins, professor, ${ }^{1}$ Richard L Berkowitz, professor, ${ }^{7}$ Susan J Gross, associate professor, ${ }^{8}$ Lorraine \\ Dugoff, associate professor, ${ }^{9}$ Sabrina D Craigo, professor, ${ }^{10}$ Ilan E Timor-Tritsch, professor, ${ }^{11}$ Stephen R \\ Carr, associate professor, ${ }^{12}$ Honor M Wolfe, associate professor, ${ }^{13}$ Mary E D’Alton, professor ${ }^{7}$
}

${ }^{1}$ Department of Obstetrics and Gynecology, University of Texas Medical Branch, 301 University Boulevard, Galveston, TX 77555 USA

${ }^{2}$ Department of Obstetrics and Gynaecology, Cambridge University, Cambridge CB2 2SW

${ }^{3}$ Department of Obstetrics and Gynaecology, Royal College of Surgeons in Ireland, Rotunda Hospital, Dublin 1, Ireland

${ }^{4}$ University of California San Francisco, San Francisco, CA 94143-013, USA

${ }^{5}$ Fetal and Women's Center of Arizona, Scottsdale, AZ 85258, USA

${ }^{6}$ William Beaumont Hospital, Royal Oak, MI 48073, USA

${ }^{7}$ Department of Obstetrics and Gynaecology, Columbia University College of Physicians and Surgeons, New York, NY 10032, USA

${ }^{8}$ Albert Einstein College of Medicine, Department of Obstetrics and Gynecology and Women's Health, Montefiore Medical Center/Albert Einstein College of Medicine, Bronx, NY 10461

${ }^{9}$ University of Colorado Health Sciences Center, Denver, CO 80262, USA

${ }^{10}$ Tufts University School of Medicine, Boston, MA 02111, USA

${ }^{11}$ Division of Ob/Gyn Ultrasound, New York University School of Medicine, New York, NY 100169196

${ }^{12}$ Brown University School of Medicine, Providence, RI 02905 , USA

${ }^{13}$ University of North Carolina Medical Center, University of North Carolina, Chapel Hill, NC 27599, USA

Correspondence to: $\mathrm{R}$ Bukowski rkbukows@utmb.edu

doi: 10.1136/bmj.39129.637917.AE

\section{ABSTRACT}

Objective To determine if first trimester fetal growth is associated with birth weight, duration of pregnancy, and the risk of delivering a small for gestational age infant.

Design Prospective cohort study of 38033 pregnancies between 1999 and 2003.

Setting 15 centres representing major regions of the United States.

Participants 976 women from the original cohort who conceived as the result of assisted reproductive technology, had a first trimester ultrasound measurement of fetal crown-rump length, and delivered live singleton infants without evidence of chromosomal or congenital abnormalities. First trimester growth was expressed as the difference between the observed and expected size of the fetus, expressed as equivalence to days of gestational age.

Main outcome measures Birth weight, duration of pregnancy, and risk of delivering a small for gestational age infant.

Results For each one day increase in the observed size of the fetus, birth weight increased by 28.2 (95\% confidence interval 14.6 to 41.2$) \mathrm{g}$. The association was substantially attenuated by adjustment for duration of pregnancy (adjusted coefficient 17.1 (6.6 to 27.5) g). Further adjustments for maternal characteristics and complications of pregnancy did not have a significant effect. The risk of delivering a small for gestational age infant decreased with increasing size in the first trimester (odds ratio for a one day increase $0.87,0.81$ to 0.94 ). The association was not materially affected by adjustment for maternal characteristics or complications of pregnancy. Conclusion Variation in birth weight may be determined, at least in part, by fetal growth in the first 12 weeks after conception through effects on timing of delivery and fetal growth velocity.

\section{INTRODUCTION}

Low birth weight infants are at increased risk of perinatal and infant death. ${ }^{1}$ Low birth weight is also associated with increased mortality in later life. ${ }^{2}$ Moreover, mothers who deliver low birth weight infants have a greater than fourfold adjusted relative risk of ischaemic heart disease. ${ }^{3}$ The factors that determine low birth weight are clearly clinically and biologically important.

Placental function in early pregnancy, as measured by circulating concentrations of placentally derived proteins in the mother's serum, is associated with risk of low birth weight. ${ }^{4}$ Moreover, animal models suggest that periconceptional undernutrition affects the timing of birth and, hence, birth weight. ${ }^{5}$ A single study has shown that fetuses that were smaller than expected in the first trimester (on the basis of menstrual history) were at increased risk of being low birth weight. ${ }^{6}$ This has been interpreted as indicating that impairment of fetal growth begins in the first trimester. However, when the expected size of the fetus is based on menstrual history, a smaller than expected fetus in early pregnancy may also reflect delayed ovulation. Other authors have suggested that a prolonged menstruationconception interval is associated with shorter duration of pregnancy and lower birth weight. ${ }^{7}$ The uncertainty in the interpretation of existing studies relates to the inherent uncertainty in using menstrual history to estimate the date of conception. We assessed the relation between discrepancy in the observed and expected size of the fetus in the first trimester and the birth weight, duration of pregnancy, and risk of delivering a small for gestational age infant among a large cohort of women with a known date of conception.

\section{METHODS}

We studied 976 women who conceived as the result of assisted reproductive technology (in vitro fertilisation or intrauterine insemination), from a previously described prospective cohort study. ${ }^{8}$ This prospective cohort study of screening for Down's syndrome took place at 15 US centres from October 1999 to December 2002. The inclusion criteria were a maternal age of 16 years or older, a singleton live fetus, and a fetal crown-rump length corresponding to a gestational age of 10 weeks 3 days to 13 weeks 6 days. We excluded women from the study if they had had 
previous measurement of nuchal translucency or if anencephaly was diagnosed in the fetus. Out of 42367 patients approached, we enrolled 38033 and $1120(3 \%)$ had conceived as a result of intrauterine insemination or in vitro fertilisation. Of these pregnancies, $14(1.3 \%)$ resulted in miscarriage, $3(0.3 \%)$ resulted in perinatal death, $2(0.2 \%)$ resulted in live births with chromosomal abnormalities, and 21 $(2.0 \%)$ resulted in live births with congenital abnormalities. Complete data on maternal characteristics, exposures, complications of pregnancy, and birth weights were available in $976(90 \%)$ of the remaining pregnancies. The final study population consisted of 976 singleton pregnancies resulting in live births without evidence of chromosomal or congenital abnormalities (table 1). All participants gave written informed consent.

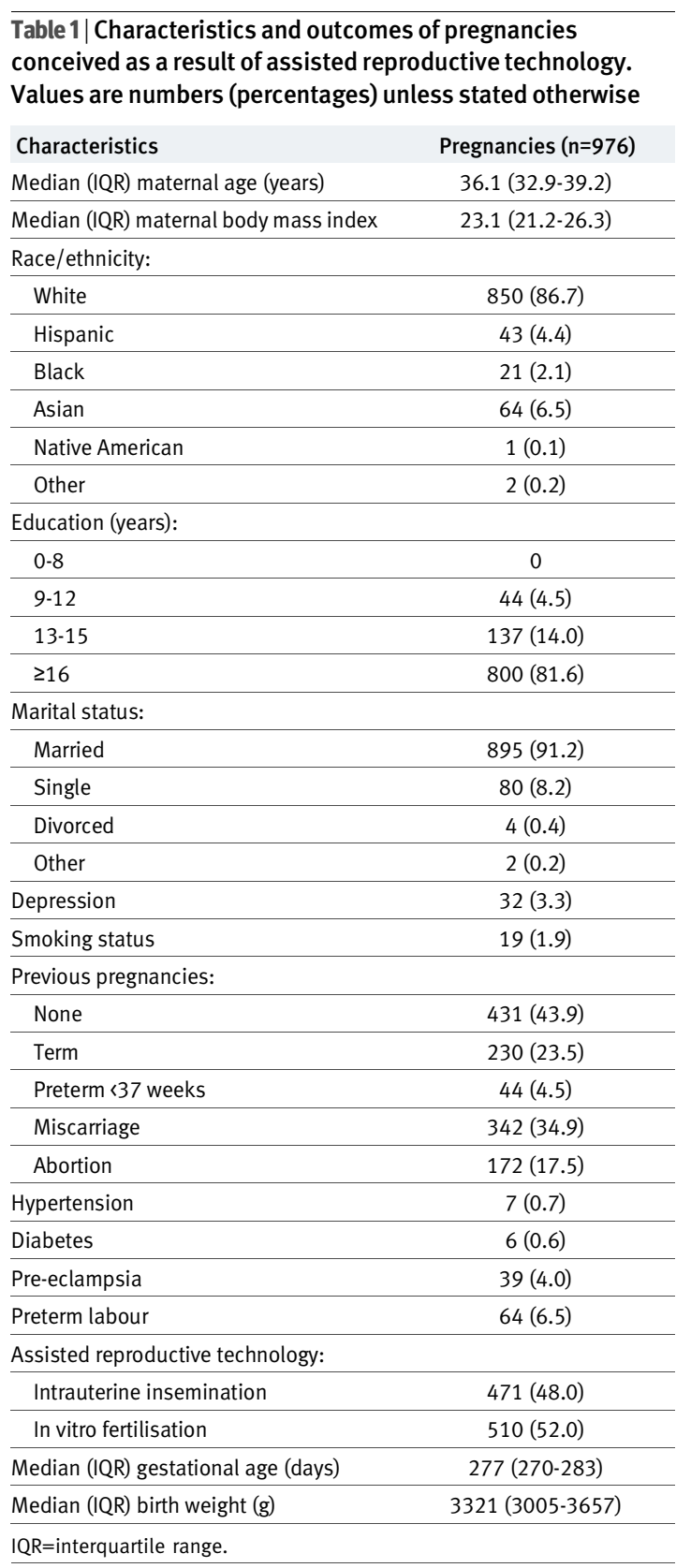

We used ultrasound to measure fetal crown-rump length before 14 weeks (between 59 and 83 days after conception). Measurement of crown-rump length has been shown to provide a precise measure of gestational age up to 14 weeks of pregnancy. Its variability in predicting menstrual age is constant in this gestational age range. ${ }^{9}$ We related the observed size of the embryo or fetus to the expected size on the basis of the date of conception, which is precisely known for pregnancies resulting from assisted reproductive technology. The time of conception is directly observed in pregnancies resulting from in vitro fertilisation. Intrauterine insemination is timed to coincide with ovulation, which is either induced or identified by measuring luteinising hormone, so the difference between time of insemination and conception is no longer than 24-36 hours. We expressed the difference between the actual and predicted crown-rump length as the difference in days of gestation $(\triangle \mathrm{GA})$ - that is, the estimated post-conception age according to crown-rump length minus the actual number of days post-conception based on the assisted reproductive method. Positive $\Delta \mathrm{GA}$ indicates a larger than expected fetus, and a negative value indicates a smaller than expected fetus.

Maternal characteristics studied were maternal age; height; weight in the first trimester; body mass index; race and ethnicity; number of previous term and preterm deliveries, miscarriages, and abortions; elevation above the sea level of the area of residence; number of completed years of education; and fetal sex. Complications of pregnancy were preterm delivery, pre-eclampsia, preterm premature rupture of the fetal membranes, preterm labour, placental abruption, and placenta praevia. We also adjusted analyses for diabetes and gestational diabetes, hypertension, smoking status and alcohol consumption, marital status, and diagnosis of depression. We defined delivery of a small for gestational age infant as birth weight below the 10th centile for gestational age. ${ }^{10}$ We used multivariable linear regression to model birth weight and multivariable logistic regression to model the risk of delivering a small for gestational age infant. We assessed linearity with fractional polynomials up to polynomials of the fourth order. ${ }^{11}$ We used the Wald test to assess the statistical significance of the change in $\Delta \mathrm{GA}$ coefficient in adjusted models. We assessed goodness of fit with the Hosmer and Lemeshow test. ${ }^{12}$ We used Stata 9.0 for analyses.

\section{RESULTS}

Birth weight varied as a linear function of $\triangle \mathrm{GA}$ : a one day increase in $\Delta \mathrm{GA}$ was associated with a $28.2 \mathrm{~g}$ increase in birth weight (table 2, fig 1). Higher order fractional polynomial models did not improve the fit. Adjustment for maternal characteristics and complications of the pregnancy had no significant effect on the association (table 2). The association between birth weight and $\Delta \mathrm{GA}$ was significantly attenuated by adjustment for duration of pregnancy (adjusted coefficient for one day increase in $\Delta \mathrm{GA} 17.1$ (95\% confidence interval 6.6 to 27.5) g). Adjustment for maternal 
characteristics and complications of pregnancy did not further attenuate the strength of this association (adjusted coefficient 15.0 (4.6 to 25.4) g). Duration of pregnancy was also proportional to $\triangle \mathrm{GA}$. A one day increase in $\triangle \mathrm{GA}$ was associated with a 0.42 day increase in duration of pregnancy (table 2). Maternal characteristics and pregnancy complications did not significantly affect the strength of this association. $\Delta \mathrm{GA}$ explained approximately $2 \%$ of the variation in birth weight, and the combination of all factors included in the full model explained $19 \%\left(\mathrm{R}^{2}=0.02\right.$; adjusted $\left.\mathrm{R}^{2}=0.19\right)$.

One hundred and thirteen (11.5\%) small for gestational age infants were born. The risk of delivering a small for gestational age infant varied inversely with first trimester growth (fig 2). A one day increase in $\Delta \mathrm{GA}$ was associated with a $13 \%$ reduction in the odds of delivering a small for gestational age infant (table 2). The relation was best described by a linear function. Higher order fractional polynomial models did not improve the fit. Adjustment for maternal characteristics and complications of pregnancy did not significantly affect the probability of delivering a small for gestational age infant (table 2). The area under the

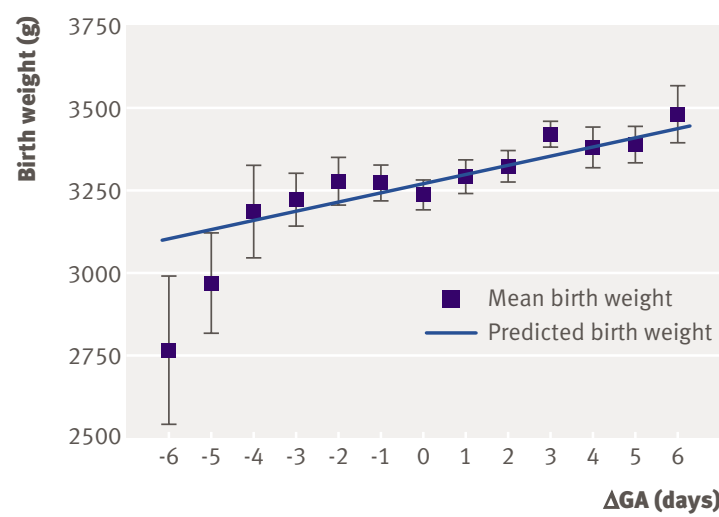

Fig 1 | Birth weight as a function of $\triangle \mathrm{GA}$ (difference between observed and expected size of fetus in first trimester of pregnancy). Mean $( \pm S E$ ) birth weights and fitted values from multivariable linear regression are plotted for each day of $\triangle \mathrm{GA}$ discrepancy receiver operating characteristics curve of $\Delta \mathrm{GA}$ as a predictor of delivery of a small for gestational age infant was 0.73 after adjustment for confounders. Gestational age at the time of ultrasonography did not have a significant effect on the associations between $\triangle \mathrm{GA}$ and birth weight $(\mathrm{P}=0.9)$ or on the risk of delivering a small for gestational age infant $(\mathrm{P}=0.3)$.

\section{DISCUSSION}

\section{First trimester growth}

Our main finding was that the size of the fetus in the first trimester of pregnancy was associated with the birth weight. Approximately half of this association was due to the effect of first trimester size on the duration of pregnancy. The clinical significance of this finding is underlined by the association between fetal growth in early pregnancy and the risk of delivering a small for gestational age infant. Before the widespread use of ultrasound, fetal growth was thought to be largely genetically determined in early pregnancy and variation in fetal growth was thought to be primarily a feature of the second half of pregnancy. ${ }^{13}$ With the widespread clinical use of ultrasound, analysis of

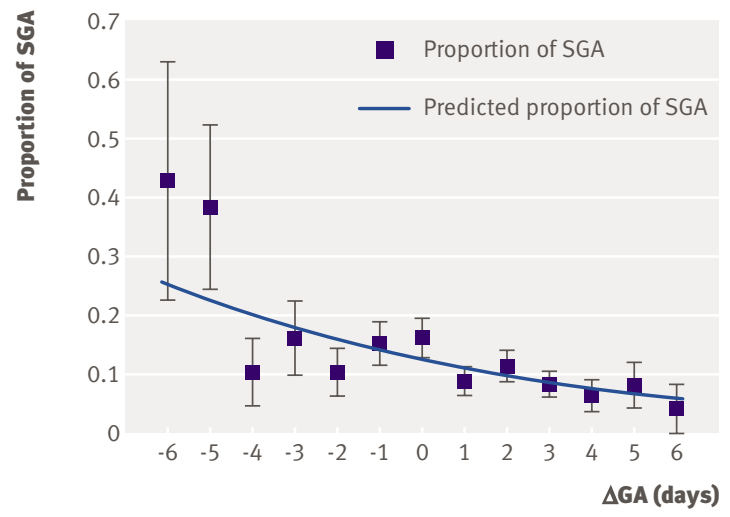

Fig 2 | Proportion of small for gestational age (SGA) neonates by size of $\triangle \mathrm{GA}$ discrepancy (difference between observed and expected size of fetus in first trimester of pregnancy). Proportion $( \pm S E)$ of SGA neonates and proportion of SGA neonates predicted by multivariable logistic regression are plotted by size of $\Delta \mathrm{GA}$ discrepancy

Table 2 | Associations between observed minus expected size of fetus in first trimester $(\Delta \mathrm{GA})$ and birth weight, duration of pregnancy, and delivery of small for gestational age infant

\begin{tabular}{|c|c|c|c|c|c|c|}
\hline \multirow[b]{2}{*}{ Outcome } & \multicolumn{2}{|l|}{ Unadjusted } & \multicolumn{2}{|c|}{$\begin{array}{l}\text { Adjusted for maternal } \\
\text { characteristics* }\end{array}$} & \multicolumn{2}{|c|}{$\begin{array}{c}\text { Adjusted for maternal characteristics } \\
\text { and pregnancy complications } \dagger\end{array}$} \\
\hline & $\begin{array}{l}\text { Coefficient/odds ratio } \neq \\
(95 \% \mathrm{Cl})\end{array}$ & $P$ value & $\begin{array}{l}\text { Coefficient/odds ratio } \neq \\
\qquad(95 \% \mathrm{Cl})\end{array}$ & P value & $\begin{array}{c}\text { Coefficient/odds ratio } \neq \\
(95 \% \mathrm{Cl})\end{array}$ & $P$ value \\
\hline Birth weight (g) & $28.2(14.9$ to 41.2$)$ & $<0.0001$ & 27.9 (14.6 to 41.2$)$ & $<0.0001$ & $27.9(15.1$ to 40.6$)$ & $<0.0001$ \\
\hline $\begin{array}{l}\text { Duration of } \\
\text { pregnancy (days) }\end{array}$ & 0.42 (0.10 to 0.73$)$ & 0.009 & $0.48(0.17$ to 0.80$)$ & 0.003 & 0.49 (0.21 to 078$)$ & 0.001 \\
\hline $\begin{array}{l}\text { Delivery of SGA } \\
\text { infant }\end{array}$ & 0.87 (0.81 to 0.94$)$ & $<0.0001$ & 0.88 (0.82 to 0.96$)$ & 0.002 & 0.90 (0.83 to 0.98$)$ & 0.013 \\
\hline
\end{tabular}

$\mathrm{SGA}=$ small for gestational age.

*Maternal age; height; weight in first trimester; body mass index; race and ethnicity; number of previous term and preterm deliveries, miscarriages, and abortions; elevation above sea level; number of completed years of education; and fetal sex.

†Preterm delivery, pre-eclampsia, preterm premature rupture of fetal membranes, preterm labour, placental abruption, and placenta praevia.

†Expressed for increase in $\triangle \mathrm{GA}$ of 1 day.

Adjustment did not result in any statistically significant change in coefficients or odds ratios (all P>0.5 compared with unadjusted coefficient); goodness of fit tests showed no evidence of lack of fit ( $P>0.5$ for all three logistic models). 


\section{WHAT IS ALREADY KNOWN ON THIS TOPIC}

The size of a fetus in the first trimester of pregnancy is associated with its birth weight

This association has been suggested to be due to delayed ovulation rather than an association with slower first trimester growth

\section{WHAT THIS STUDY ADDS}

Fetal growth in the first trimester of pregnancy was strongly associated with birth weight in pregnancies with a known date of conception

Approximately half of this association was due to the effect of first trimester size on the duration of pregnancy and half on fetal growth in later pregnancy

The risk of delivering a small for gestational age infant is inversely related to first trimester growth length may simply have been at an earlier stage of development. For example, if implantation and early embryonic development were delayed for a given assumed date of conception, the fetus would have a smaller than expected crown-rump length. However, delayed implantation would lead to both smaller than expected crown-rump length and apparently longer duration of pregnancy. In fact, we saw exactly the opposite association, and delayed implantation cannot explain the observed associations.

\section{Implications}

Measurement with ultrasound is recognised to be a more accurate method of estimating gestational age in the first trimester of pregnancy than menstrual dating, owing to errors in the assumed date of conception based on the last menstrual period. Therefore, for the purposes of intervention or screening, gestational age is adjusted on the basis of ultrasound criteria. However, our findings show that discrepancy in gestational age, where the time of conception is known, is related to the risk of delivering a small for gestational age infant. In women with a certain date of last menstrual period and a regular cycle and in women who conceive through assisted reproductive technology, pregnancies where the crown-rump length is smaller than expected may thus benefit from closer monitoring, especially for abnormalities of fetal growth.

These observations add to a growing body of evidence that duration of pregnancy and complications of late pregnancy may be the ultimate consequence of conditions in the very earliest stages of pregnancy. ${ }^{1516}$ Adverse outcome of pregnancy in a considerable proportion of women is likely to be determined before their first prenatal visit. These findings underline the importance of detailed study of the periconceptional period and first trimester of pregnancy when assessing factors that influence the risk of adverse outcome and in the development of predictive tests.

Contributors: RB and GCSS conceptualised and designed the study, analysed and interpreted the data, drafted the paper, and approved the version to be published. All authors participated in analysis and interpretation of data, revised the manuscript critically for important intellectual content, and approved the version to be published. RB is the guarantor.

Funding: The FASTER trial was supported by grant from the National Institutes of Health and National Institute of Child Health and Human Development (RO1 HD 38625). The funders had no role in study design; data collection and analysis; decision to publish; or preparation, review, and approval of the manuscript.

Competing interests: None declared.

Ethical approval: Institutional review boards in all centres gave approval. cies with a fetal abnormality, and this cannot explain the observed association.

\section{Birth weight and duration of pregnancy}

Strong and statistically significant associations between $\triangle \mathrm{GA}$ and both birth weight and the risk of delivering a small for gestational age infant persisted after adjustment for duration of pregnancy, a wide range of maternal characteristics, and complications of pregnancy. This suggests a major, independent effect of early pregnancy growth on both outcomes. Fetuses with a smaller than expected crown-rump
1 Kramer MS. Determinants of low birth weight: methodological assessment and meta-analysis. Bull World Health Organ 1987;65:663-737.

2 Barker DJP. Mothers, babies and disease in later life. London: BMJ Publishing Group, 1994.

3 Smith GC, Pell IP, Walsh D. Pregnancy complications and maternal risk of ischaemic heart disease: a retrospective cohort study of 129,290 births. Lancet 2001;357:2002-6.

4 Smith GC, Stenhouse El, Crossley JA, Aitken DA, Cameron AD, Connor JM. Early-pregnancy origins of low birth weight. Nature 2002;417:916.

5 Bloomfield FH, Oliver MH, Hawkins P, Campbell M, Phillips DJ, Gluckman PD, et al. A periconceptional nutritional origin for noninfectious preterm birth. Science 2003;300:606 
6 Smith GC, Smith MF, McNay MB, Fleming JE. First-trimester growth and the risk of low birth weight. N Engl J Med 1998;339:1817-22.

7 Gardosi J, Francis A. Early pregnancy predictors of preterm birth: the role of a prolonged menstruation-conception interval. BJOG 2000;107:228-37.

8 Malone FD, Canick JA, Ball RH, Nyberg DA, Comstock CH, Bukowski R, et al. First-trimester or second-trimester screening, or both, for Down's syndrome. N Engl J Med 2005;353:2001-11.

9 Hadlock FP, Shah YP, Kanon DJ, Lindsey JV. Fetal crown-rump length: reevaluation of relation to menstrual age ( 5 -18 weeks) with highresolution real-time US. Radiology 1992;182:501-5.

10 Alexander GR, Himes JH, Kaufman RB, Mor J, Kogan M. A United States national reference for fetal growth. Obstet Gynecol 1996;87:163-8

11 Royston P, Altman DG. Regression using fractional polynomials of continuous covariates-parsimonious parametric modeling. I $R$ Stat Soc Ser C Appl Stat 1994;43:429-67.
12 Hosmer DW, Lemeshow S. Goodness of fit tests for the multiple logistic regression-model. Communications in Statistics Part ATheory and Methods 1980;9:1043-69.

13 Gluckman PD, Liggins GC. Regulation of fetal growth. In: Beard RW Nathanielsz PW, eds. Fetal physiology and medicine: basis of perinatology. New York: Dekker, 1984:511-58.

14 Bahado-Singh RO, Lynch L, Deren O, Morroti R, Copel JA, Mahoney MJ, et al. First-trimester growth restriction and fetal aneuploidy: the effect of type of aneuploidy and gestational age. Am J Obstet Gynecol 1997;176:976-80.

15 Salomon LJ, Cavicchioni O, Bernard JP, Duyme M, Ville Y. Growth discrepancy in twins in the first trimester of pregnancy. Ultrasound Obstet Gynecol 2005;26:512-6.

16 Smith GC. First trimester origins of fetal growth impairment. Semin Perinatol 2004;28:41-50

Accepted: 1 February 2007 